\title{
Interstitial lung disease in systemic sclerosis: where do we stand?
}

\author{
Susanna Cappelli ${ }^{1}$, Silvia Bellando Randone ${ }^{1}$, Gianna Camiciottoli², \\ Amato De Paulis ${ }^{3}$, Serena Guiducci ${ }^{1}$ and Marco Matucci-Cerinic ${ }^{1}$
}

Affiliations: 'Dept of Experimental and Clinical Medicine, Division of Rheumatology AOUC, School of Medicine, University of Florence, Florence, Italy. ${ }^{2}$ Dept of Experimental and Clinical Medicine, Division of Pneumology and Lung Physiopathology AOUC, School of Medicine, University of Florence, Florence, Italy. ${ }^{3}$ Dept of Translational Medical Sciences and Center for Basic and Clinical Immunology Research (CISI), University of Naples Federico II, Naples, Italy.

Correspondence: Susanna Cappelli, Dept of Experimental and Clinical Medicine, Division of Rheumatology AOUC, School of Medicine, University of Florence, Viale Pieraccini 18, 50139 Florence, Italy.

E-mail: susicappelli82ayahoo.it

ABSTRACT Interstitial lung disease (ILD) is common in systemic sclerosis (SSc) patients and despite recent advances in the treatment is, at present, the major cause of death. Today, an early diagnosis of ILD is possible, and is mandatory to improve the prognosis of the disease.

Pulmonary function tests and high-resolution computed tomography remain the mainstay for the diagnosis of SSc-ILD, but there is a growing interest in lung ultrasound. Recently, the correlation between severity of fibrosis and some peripheral blood biomarkers has been described.

Nonselective immunosuppressors are still the main treatment for ILD, with cyclophosphamide (CYC) most widely used to obtain remission. Novel therapies towards specific molecular and cellular targets have been suggested; in particular, rituximab (RTX) has shown promising results, but further research is needed. It is of paramount importance to define the severity of the disease and the risk of progression in order to define the need for treatment and the treatment intensity. We propose the division of the treatment strategies at our disposal to induce remission into three categories: high intensity (haematopoietic stem cell transplantation), medium intensity (CYC and RTX) and low intensity (azathioprine (AZA) and mycophenolate mofetil (MMF)). After obtaining remission, maintenance treatment with AZA or MMF should be started.

In this review we explore new advances in the pathogenesis, diagnosis and treatment of SSc-ILD.

\section{-}

@ERSpublications

Early diagnosis of ILD is possible, and is mandatory to improve the prognosis of the disease http://ow.ly/P28JH

The relevance of interstitial lung disease in systemic sclerosis

Pulmonary disease in systemic sclerosis (SSc) mainly comprises interstitial lung disease (ILD) and pulmonary arterial hypertension (PAH). Over the past 40 years the SSc mortality rate has not changed significantly [1]. Nevertheless, while the frequency of deaths due to renal crisis has significantly decreased from $42 \%$ to $6 \%$, the proportion of deaths due to ILD and PAH has increased [2]. In fact, ILD and $\mathrm{PAH}$ are the two main causes of death in SSc, accounting for 33\% and $28 \%$ of deaths, respectively [2]. A European Scleroderma Trials and Research group (EUSTAR) analysis revealed, in a cohort of 3656 SSc patients, that ILD is present in 53\% of cases with diffuse cutaneous SSc and in 35\% of cases with limited

Received: March 282015 | Accepted after revision: April 272015

Conflict of interest: None declared.

Provenance: Publication of this peer-reviewed article was sponsored by F. Hoffman-La Roche Ltd, Basel, Switzerland (principal sponsor, European Respiratory Review issue 137).

Copyright OERS 2015. ERR articles are open access and distributed under the terms of the Creative Commons Attribution Non-Commercial Licence 4.0. 
cutaneous SSc [3]. Cumulative survival of SSc patients from diagnosis is $84.1 \%$ at 5 years and $74.9 \%$ at 10 years [4]. The reported survival of SSc-ILD patients is similar to that of patients without ILD at 5 years while it is significantly lower at 10 years. In fact, the survival of SSc-ILD patients is reported to be 29-69\% at 10 years [5]. In early autopsy studies, up to $100 \%$ of patients have parenchymal involvement [4], as many as $90 \%$ of patients show interstitial abnormalities on high-resolution computed tomography (HRCT) [6], and 40-75\% have changes in pulmonary function tests (PFTs) [7]. Considering the frequency of lung involvement in SSc and its impact on the prognosis, it is important to recognise patients with ILD early and treat them appropriately.

\section{Pathogenesis}

The pathogenesis of SSc-ILD is not yet fully understood. Three steps are considered to be involved: 1) persistent and repeated bouts of injury to endothelial cells, 2) activation of innate and adaptive immunity and 3) fibroblast recruitment/activation, which results in accumulation of extracellular matrix and scarring [8]. Still, the exact pathways and mechanisms are not clear and have been the object of recent studies. A pivotal role seems to be played by transforming growth factor (TGF)- $\beta$ which is secreted by platelets, monocytes/ macrophages, T-cells and fibroblasts. The binding of TGF- $\beta$ to its receptor activates Smad-dependent and Smad-independent pathways, including the mitogen-activated protein kinases p38 and c-Jun N-terminal kinase, the lipid kinase phosphoinositol-3-kinase, the tyrosine kinase c-ABL, and the Rho-associated coiled-coil containing protein kinase [9]. Target genes of Smad-dependent TGF- $\beta$ signalling are type I collagen, plasminogen activator inhibitor, $\alpha$-smooth muscle actin and connective tissue growth factor. Toll-like receptor (TLR) 4 is widely recognised as central to the innate immune response to Gram-negative bacteria, but it can also be activated by endogenous ligands generated by cellular injury, the autoimmune response and oxidative stress. In skin and lung biopsies of SSc patients, increased expression of TLR4 has been demonstrated. TLR4 activation potentiates TGF- $\beta$ signalling and suppresses antifibrotic microRNA [10].

Telocytes are a peculiar type of stromal cell, which may have a role in the regulation of tissue homeostasis. A recent study has shown loss of telocytes in the gastric wall, myocardium and lung of SSc patients, suggesting that this loss may be implicated in the pathogenesis of fibrosis [11]. Recently, elevated levels of interleukin (IL)-33 have been correlated with the severity of skin and lung fibrosis [12]. Another protein that may be involved in the pathogenesis of SSc-ILD is caveolin-1. It interferes with the TGF- $\beta$ pathway since it inhibits Smad 3 phosphorylation and increases the endocytosis and degradation of TGF- $\beta$ ligand-receptor complexes. In SSc patients, low levels of surface caveolin-1 have been detected [13]. Thymosin $\beta-4$ is a small ubiquitin protein, which acts by promoting cell motility and cell adhesion, inhibiting apoptosis, and downregulating inflammation. Lower levels of thymosin $\beta-4$ in the bronchoalveolar lavage of SSc-ILD patients have been correlated with disease progression [14]. In recent years, some genetic studies have shown increased expression of the TGF- $\beta$ response including fibrosis-associated genes and myofibroblast markers $[15,16]$. An analysis of gene expression in skin biopsies identified some transcripts that correlate with the severity of SSc-ILD. Plasma levels of two proteins encoded by these genes, CCL2 and soluble P-selectin glycoprotein ligand 1, correlate with forced vital capacity (FVC) in PFTs, suggesting their possible use as biomarkers [17].

Epigenetic mechanisms may also play a role in the pathogenesis of SSc-ILD. Alterations in DNA methylation and histone modifications have been described in SSc-ILD patients [18]. The role of epigenetic alterations has not been widely investigated and it represents a challenging research area.

\section{Identification and follow-up of patients with SSC-ILD}

The history of patients with SSc-ILD usually reveals the insidious onset of exertional dyspnoea and a nonproductive cough. Bilateral basilar dry inspiratory crackles are usually present at lung auscultation. Virtually, all SSc-ILD patients are positive for antinuclear antibodies, which are frequently accompanied by SSc-specific antibodies, anti-topoisomerase I (anti-Topo I) and anti-Th/To and more rarely by anti-centromere antibodies [19]. PFTs reveal a restrictive pattern, mainly due to parenchymal involvement, with reduced forced expiratory volume in $1 \mathrm{~s}$ (FEV1) and FVC, and a normal or slightly increased FEV1/FVC ratio. Restrictive lung disease from severe thoracic cutaneous involvement has been reported. Airway disease, although described, is rare in SSc as compared with other connective tissue diseases [20]. Diffusing capacity of the lung for carbon monoxide (DLCO) is the most important functional test due to its ability to investigate the interstitial space between alveolar and endothelial surfaces as well as the integrity of the lung vascular bed. In patients with SSc, it can be reduced either in parenchymal fibrosis or in vascular abnormalities of pulmonary hypertension. Recently a rapid decline of DLCO has been identified as the single most significant marker of poor outcome [5]. Furthermore, it has been shown that DLCO provides the best estimate of the extent of ILD on HRCT [21]. Concerning imaging techniques, chest radiography has a low sensitivity and specificity for detecting parenchymal involvement. By contrast, a good specificity has been demonstrated in pulmonary hypertension, where typical findings are the enlargement of right pulmonary artery, loss of peripheral vasculature and filling of the retrosternal space by the hypertrophic right ventricle [22]. For the diagnosis of 
SSc-ILD, HRCT is mandatory. The most frequent HRCT pattern is nonspecific interstitial pneumonia (NSIP) [23], with a greater proportion of ground-glass opacities (GGOs) and a lower proportion of coarse reticulation. However, a usual interstitial pneumonia (UIP) pattern, characterised by honeycombing and traction bronchiectasis, may also be seen. Since the HRCT pattern predicts the underlying histopathology well, a lung biopsy is not generally necessary. The reversal of HRCT modifications is rarely achieved. GGO is commonly associated with irreversible disease. In fact, only 5\% of patients with GGO and nonfibrotic interstitial opacities show an improvement of HRCT findings. Thus, in SSc, GGO may represent fibrosis in many cases [24]. Over time, the radiographic progression involves the replacement of GGOs with honeycombing/traction bronchiectasis and/or bronchiolectasis [25].

Recently, the issue of X-ray exposure in SSc patients has been raised [26, 27]. For this reason, the use of a 9 -slice HRCT protocol has been evaluated in order to reduce the radiation dose and has shown an accuracy of over $90 \%$ when compared with standard HRCT [27]. Quantitative CT densitometry has been proposed as a method of providing a sensitive assessment of lung structure for monitoring parenchymal damage [28] and a good correlation with PFTs has been shown [28-30]. In recent years, there has been a growing interest in lung ultrasound (LUS) as a fast and noninvasive method with no risk of radiation exposure [26, 31]. The ultrasound feature of ILD involves the detection and quantification of the lung comet tail signs (B-lines) that originate from thickened interlobular septa. The number of B-lines has shown a good correlation with lung involvement on HRCT [32]. Nevertheless, the role of LUS in reducing the number of HRCTs in the follow-up of patients awaits further validation [33]. LUS cannot replace HRCT for diagnosis and for a complete evaluation of lung involvement. We propose an algorithm, reflecting our experience, for the diagnosis and follow-up of SSc-ILD that may include the use of LUS (fig. 1). This algorithm suggests that SSc patients should perform PFTs, HRCT and LUS to reach a diagnosis. Patients without lung involvement should then be monitored every 1-2 years (depending on the presence of risk factors) using PFTs with DLCO, which are easy to perform, repeatable, reproducible, noninvasive and not expensive, and, if available, using LUS. If ILD is suspected from these examinations patients should move on to HRCT. Patients with evidence of ILD-SSc should perform PFTs with DLCO every 6 months and, if available, LUS; HRCT should be performed if progression of lung disease is suspected.

\section{The outcome and its predictors}

Unlike the idiopathic interstitial pneumonias, survival of patients with NSIP does not appear to differ from that of patients with UIP [5]. Severe restrictive lung disease (defined by a FVC of $\leqslant 50 \%$ predicted) has been reported to occur in $13 \%$ of patients [7]. Patients who develop severe ILD tend to have a progressive decline in lung function within the first 2 years of disease [7]. It is of paramount importance to identify those patients who will develop severe ILD. Many demographic, clinical and serological features associated with disease progression have been described: male sex, black ethnicity/race, older age, early disease, and the presence of anti-Topo I antibodies [5, 34-37].

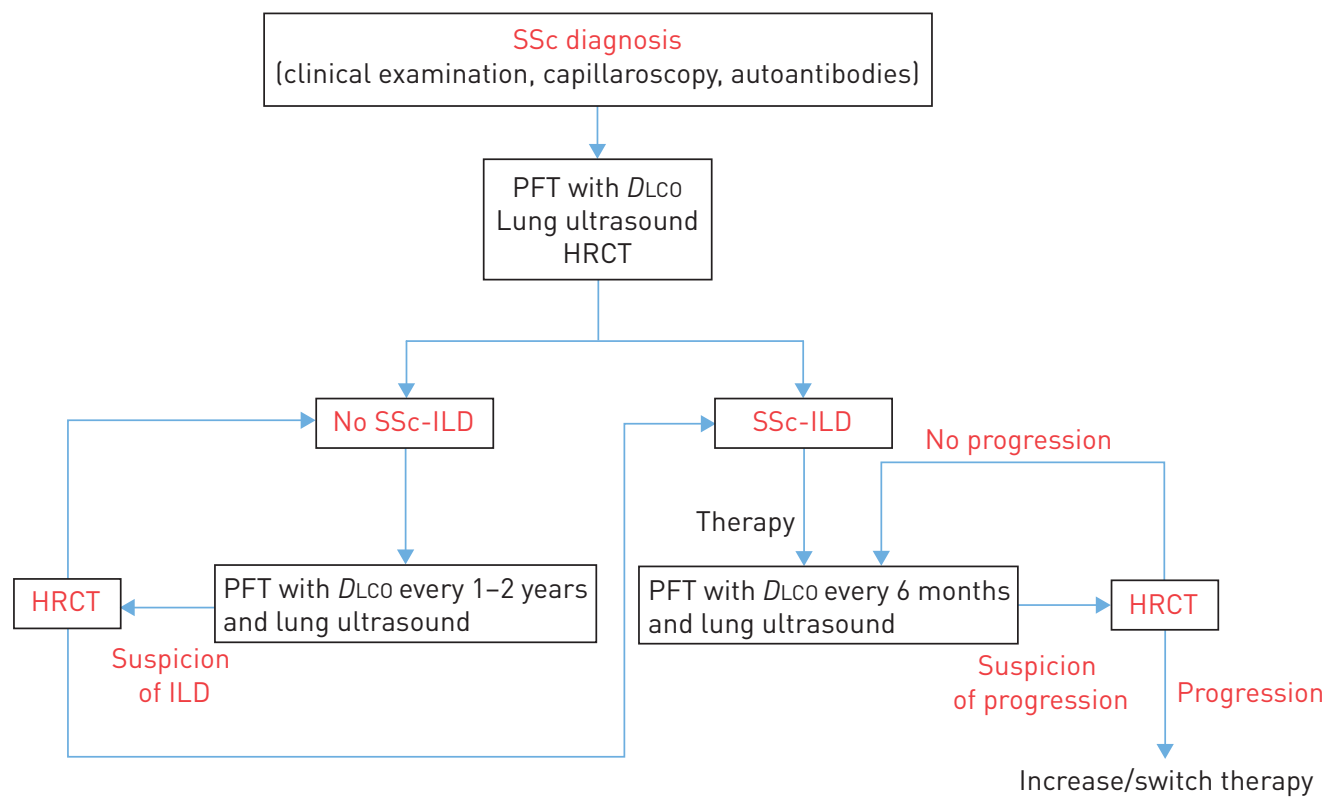

FIGURE 1 Algorithm for diagnosis and follow-up of systemic sclerosis-interstitial lung disease (SSc-ILD). PFT: pulmonary function test; DLCO: diffusing capacity of the lung for carbon monoxide; HRCT: high-resolution computed tomography. 
Recently, an algorithm based on combined evaluation of HRCT and PFTs has been proposed to differentiate patients with extensive and limited lung disease. Patients with $>20 \%$ HRCT abnormalities are considered to have extensive lung disease and those with $<20 \%$ to have with limited disease. If HRCT evaluation is inconclusive, patients are considered to be affected by extensive lung disease if FVC $<70 \%$ predicted and by limited lung disease if $\mathrm{FVC} \geqslant 70 \%$ predicted. Patients with extensive lung disease have strikingly higher mortality and faster deterioration of lung function [38]. A meta-analysis of 26 studies identified male sex, disease extent on HRCT, presence of honeycombing, elevated Krebs von den Lungen (KL-6) values and increased alveolar epithelial permeability as predictors of both mortality and ILD progression in unadjusted analysis, while disease extent on the HRCT scan was the only variable that independently predicted both mortality and ILD progression [39]. Recently, a loss of density on capillaroscopy was shown to be associated with worse FVC and DLCO [40] and biomarkers that correlate with the severity of fibrosis have been proposed: elevated levels of chitinase [41], tenascin C [42], growth differentiation protein 15 [43], cartilage oligomeric matrix protein, alveolar epithelial cell antigen, KL-6 [44] and the chemokine CXCL4 [45]. Serum IL-6 predicted early disease progression and IL-6 values $>767 \mathrm{pg} \cdot \mathrm{mL}^{-1}$ were predictive of risk of death within the first 30 months in patients with mild SSc-ILD [46].

\section{Treatment}

Since inflammation precedes and leads to fibrosis, and it is believed that only the inflammation is in part reversible, early treatment is mandatory. Nevertheless, fibrosis is generally the predominant abnormality in SSc-ILD. In a large histopathological series, inflammatory abnormalities were present in $<20 \%$ of cases [5]. At present, no treatment can reverse lung fibrosis, and therefore the prevention of disease progression is still a more realistic goal than disease regression.

When faced with a patient with SSc-ILD, it is of paramount importance to understand the risk of progression in order to decide if therapy should be started and the level of intensity of the treatment. Therefore, the physician should make a profile of the patient that includes the stage, the activity and the severity of the disease. Even if there are no studies showing that extensive disease, as defined by GoH et al. [38], is a criterion to start treatment, experts will treat patients with extensive disease, while a "wait and watch" approach is not infrequently chosen when managing patients with mild disease. SSc patients with evidence of ongoing disease progression, based on pulmonary function decline or radiographic deterioration, need treatment independent of disease extent. Features that may predict progression (e.g. early disease, tendon friction rub, anti-Topo I positivity and high IL-6 levels) may in future be considered when deciding whether or not to start an immunosuppressive therapy.

\section{Nonselective immunosuppressors}

Nonselective immunosuppressors are still the most widely used treatment in SSc-ILD. Therapy for ILD consists of an induction and a maintenance phase (fig. 2).

After two double-blind [47, 48] and one unblinded [49] randomised controlled trials (RCTs), in which patients treated with cyclophosphamide (CYC) showed either a significant increase or a trend towards an increase in FVC [47-49], CYC is the drug most used as an induction regimen. Results from the Scleroderma Lung Study I showed that the beneficial effects of oral CYC persisted or increased for several months after stopping the therapy, but were no longer apparent after 12 months; suggesting that maintenance treatment after CYC therapy is needed [47]. When using CYC, toxicity is an issue; in the short term this includes leukopenia and infections, while long-term effects include infertility, bone marrow toxicity and carcinogenesis. The use of intermittent intravenous pulses reduces the toxicity [50]. There is still no agreement on dose, duration and frequency of CYC pulses [48, 51-53]. We suggest the use of monthly pulses at a dose of $1 \mathrm{~g} \cdot \mathrm{m}^{-2}$ for 6-12 months. The dose of each infusion and the duration of the therapy can be reduced according to age and comorbidity.

Recently, the use of mycophenolate mofetil (MMF) in the treatment of SSc-ILD has produced great interest. To date, only observational studies on the efficacy of MMF have been performed. A recent meta-analysis, encompassing one prospective [54] and four retrospective studies [55-58], evaluated the safety and efficacy of MMF and mycophenolate sodium [59]. The results of this meta-analysis show that mycophenolate is a safe therapeutic agent and its administration has been linked with stabilisation of lung functional parameters. A prospective study in which patients treated with MMF were matched 1:1 with patients treated with CYC, showed a deterioration of lung HRCT at 2 years after MMF treatment but not after CYC, despite the fact that patients treated with CYC had more extensive ILD at baseline [60]. While awaiting the results of a multicentre randomised clinical trial, the Scleroderma Lung Study II, which aims to compare the functional effect of MMF with oral CYC in patients with SSc-ILD, MMF is generally used as a maintenance treatment after CYC and is only occasionally employed as an induction treatment [61]. 


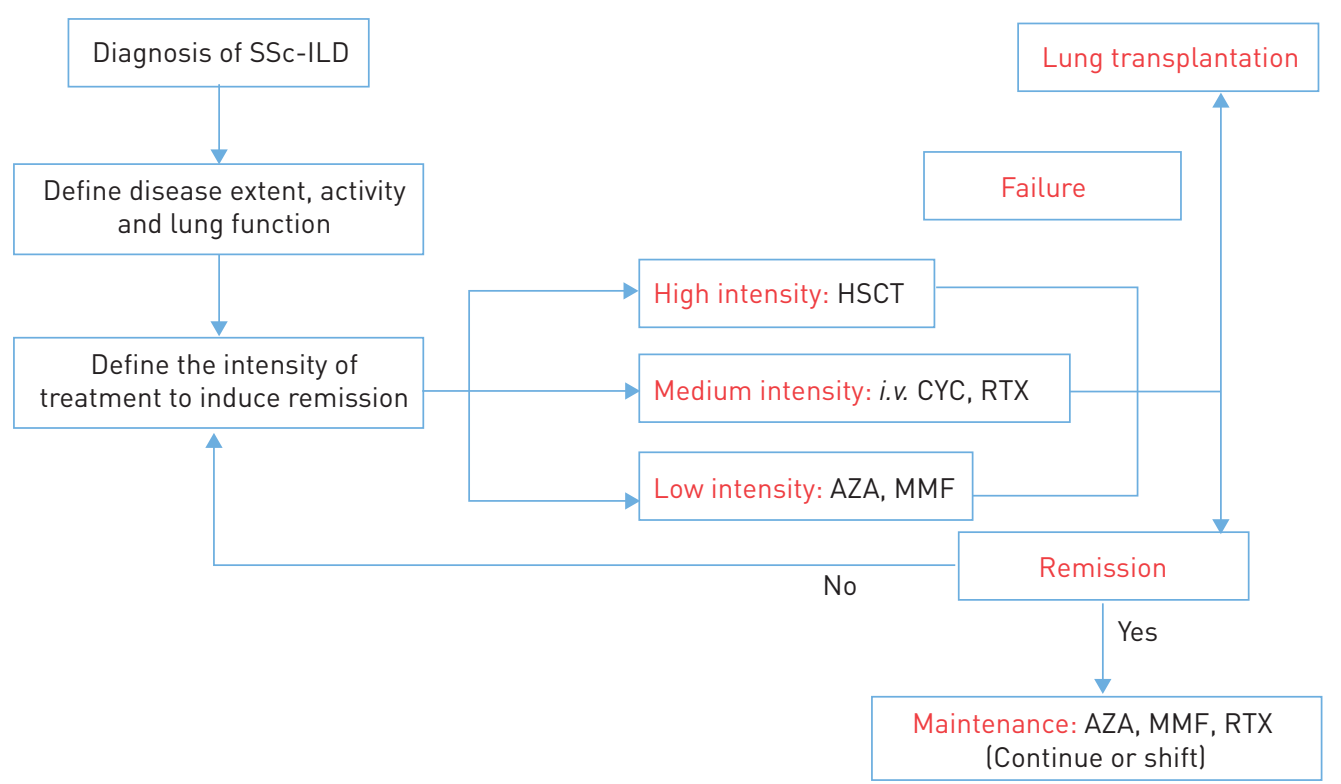

FIGURE 2 Algorithm to induce remission in systemic sclerosis-interstitial lung disease (SSc-ILD). HSCT: haematopoietic stem cell transplantation; CYC: cyclophosphamide; RTX: rituximab; AZA: azathioprine; MMF: mycophenolate mofetil.

In a RCT, azathioprine (AZA) has been compared with CYC as first line treatment. Unlike patients treated with CYC who showed a trend towards FVC improvement, in patients receiving AZA a significant deterioration of both FVC and DLCO was observed after 10 months of follow-up [49]. The results of observational studies are encouraging concerning the use of AZA as a maintenance agent, as AZA stabilised lung function in most patients $[51,62,63]$. In view of these results we usually do not recommend the use of AZA as first-line agent but mainly as a maintenance treatment after CYC. Currently, there are no parameters to guide the choice between AZA and MMF as the maintenance treatment. In a recent study patients with deterioration of lung function after CYC received MMF and those with improvement or stabilisation were treated with AZA. Most of patients unresponsive to CYC also worsened under MMF [51].

Corticosteroids are often used in SSc patients, predominantly in combination with other immunosuppressive treatments since monotherapy with corticosteroids is generally not effective. Corticosteroid pulses have been used in association with CYC in the treatment of ILD-SSc with favourable results $[64,65]$. It is important to remember that the chronic use of corticosteroids at a medium-high dose $\left(>15 \mathrm{mg} \mathrm{day}^{-1}\right.$ ) has been associated with a higher risk of developing a scleroderma renal crisis (SRC), particularly in patients with early diffuse disease $[66,67]$.

\section{Biological immunotherapies}

In terms of biological treatment, interesting results on the use of rituximab (RTX) have been published. A few years ago, a pilot study randomised 14 diffuse cutaneous SSc patients to receive either RTX plus standard therapy or standard therapy alone. Two cycles of RTX were administered (at baseline and 24 weeks). Each cycle consisted of four weekly RTX infusions $\left(375 \mathrm{mg} \cdot \mathrm{m}^{-2} \cdot\right.$ week $\left.^{-1}\right)$. After 1 year of follow-up, patients treated with RTX showed a significant improvement in FVC, DLCO and modified Rodnan skin score (mRss) [68]. Two further cycles of RTX, at an interval of 6 months, were then administered to the patients in the RTX group. After 2 years of follow-up, a significant improvement in pulmonary function (FVC: $+9 \%, \mathrm{p}<0.0001$; DLCO: $+10.88 \%, \mathrm{p}<0.001$ ) and skin involvement was observed (mRss: $-8.63, \mathrm{p}<0.0001$ ) [69]. In 2014, a case-control analysis, carried out by the EUSTAR group, was published. 63 patients treated with RTX were matched with control patients from the EUSTAR database not treated with RTX. In SSc-ILD patients RTX significantly prevented further decline of FVC compared with matched controls $(p=0.02)$ [70]. Although the efficacy of RTX is awaiting validation in a RCT, these results are encouraging and we consider RTX as a valid treatment option in patients who do not tolerate CYC or who have a contraindication for it. RTX may also be used after CYC instead of starting maintenance with MMF or AZA, particularly if the patient fails to response to CYC.

As for other biological therapies (abatacept, tocilizumab and anti-tumour necrosis factor (TNF)- $\alpha$ ), there are only a few studies evaluating their efficacy in SSc-ILD. In particular, we do not recommend the use of anti-TNF- $\alpha$ since its effects on fibrosis are not clear [71]. 


\section{Antifibrotic treatment}

In SSc, no antifibrotic drug has shown a real efficacy in prevention and treatment of fibrosis. The tyrosine kinase inhibitor, imatinib mesilate, blocks the pro-fibrotic c-Abl kinase, an important downstream signalling molecule of TGF- $\beta$ [72]. In two single-arm, open label, phase I/IIa and IIa clinical trials, patients treated with imatinib showed a significant reduction of mRss. A statistically significant improvement in lung function was only shown in one of the studies. In both studies adverse events (facial/ lower extremity oedema, fatigue, gastrointestinal and musculoskeletal complaints) were common [73, 74]. In a proof-of-concept, double-blind, RCT, imatinib was interrupted after 6 months due to its poor tolerability [75]. Only four out of the 10 active diffuse cutaneous SSc patients enrolled were able to complete the 6 months of treatment. In this study, imatinib was used at a dose of $400 \mathrm{mg}$ twice a day; however, when the dose was reduced to $200 \mathrm{mg}$ daily it was also poorly tolerated. By contrast, in a very recent study in which patients received $200 \mathrm{mg}$ daily, imatinib was well tolerated [76]. Imatinib has been evaluated in a RCT in patients with idiopathic pulmonary fibrosis. In this study imatinib at a dose of $600 \mathrm{mg}$ daily did not affect either survival or lung function. Serious adverse events were not more frequent in the imatinib group than in the placebo group [77]. Considering the lack of clear evidence of efficacy and the quite common side-effects, imatinib is rarely used in clinical practice.

Thalidomide has shown antifibrotic properties on interstitial lung fibrosis in in vitro studies of human fetal lung fibroblasts and in vivo studies of a mouse model of ILD generated by bleomycin injection [78]. Further studies are needed in order to define if it can represent a treatment option for SSc-ILD patients.

\section{Immunoglobulins}

The use of intravenous immunoglobulins has been evaluated in a randomised double-blind placebo-controlled trial and in some open-label studies, in which an improvement of skin involvement was observed [79-82]. In these studies the effects on ILD were not evaluated. The case of a SSc patient with myositis and early-stage ILD was recently described, in which, after i.v. immunoglobulins, the regression of GGOs, septal thickenings and a full recovery of lung function occurred [83].

\section{Haematopoietic stem cell transplantation}

In the past decade, intense immunosuppression followed by haematopoietic stem cell transplantation (HSCT) has emerged as a new therapeutic procedure for patients affected by severe diffuse cutaneous SSc that is refractory to conventional treatments [84]. Recently, the results of the Autologous Stem Cell Transplantation International Scleroderma Trial (ASTIS), a phase III, multicentre, randomised study, were published. In this trial, HSCT was compared, in terms of efficacy and safety, with 12 successive monthly i.v. pulses of CYC. In terms of effects on lung function, patients undergoing HSCT showed a significant improvement of both FVC and total lung capacity, while no significant differences in terms of DLCO were observed. The number of events, defined as death or persistent major organ failure, was recorded in the two groups. Patients undergoing HSCT showed a higher number of events in the first year: 13 (16.5\%) events, including eight treatment-related deaths, in the HSCT group versus eight (10.4\%) events, with no treatment-related deaths, in the control group. But the total of number of events, observed during a median follow-up of 5.8 years, was higher in the CYC treated group: 22 events in the HSCT group (19 deaths and three irreversible organ failures) and 31 events in the CYC group (23 deaths and eight irreversible organ failures). Therefore, HSCT was associated with increased treatment-related mortality in the first year after treatment, but it conferred a significant long-term event-free survival benefit and may improve lung function [85]. Nevertheless, before deciding on transplantation the high treatment-related risk of death should always be considered.

\section{Lung transplantation}

Lung transplantation is a life-saving option for SSc-ILD patients who are not responsive to medical therapy. Unfortunately lung transplantation is not always possible due to the involvement of other organs.

The short- and intermediate-term survival of SSc-ILD patients post-lung transplantation are similar to those seen in idiopathic pulmonary fibrosis [86]. Considering the main studies on lung and heart-lung transplantation an overall survival of $52-80 \%$ at 2 years is reported [87-90]. In a recent retrospective analysis a survival rate of $59 \%$ is reported at 3 years [91]. The study with the best survival outcomes had more stringent exclusion criteria [89].There are no official specific contraindications for lung or heart-lung transplantation in SSc. Independent of the disease that leads to transplantation, absolute contraindications are: untreatable organ failure other than lung (especially creatinine clearance $<50 \mathrm{~mL} \cdot \mathrm{min}^{-1} / 1.73 \mathrm{~m}^{-2}$ ), non-curable chronic extrapulmonary infections, active or recent neoplasia (less than 2 years), active smoking or active substance addition, uncontrolled psychiatric disorders, major spinal/thoracic deformity and degenerative neuromuscular diseases, documented non-adherence with medical therapy, and body mass index $<15 \mathrm{~kg} \cdot \mathrm{m}^{-2}$. Recently, specific SSc contraindications have been proposed. They include: uncontrolled active inflammatory myopathy, active digital ulcers, severe gastrointestinal involvement, 
cardiac arrhythmias, unstable renal function in the past 3 months (except in the case of acute functional renal failure related to right ventricle dysfunction), an interval of $<3$ years between SRC and transplantation, and increased risk of SRC (significant skin breakdown from severe cutaneous disease, diffuse SSc evolving for $<3$ years since the first non-Raynaud symptoms) [91].

\section{Conclusions}

The early diagnosis of ILD is possible and should be achieved by every specialist. It is not only necessary to get an early diagnosis, but also to define the severity of the disease and try to predict patients' prognosis. In this effort, risk factors for evolution should be checked in every patient. According to the severity of the disease the physician should define how aggressive the therapy should be. Early treatment is mandatory to achieve a better prognosis. To obtain remission, that is to stop disease progression and, if possible, to reverse some disease-related changes, is the aim of every treatment strategy. Patients should be evaluated regularly in order to define if remission has been reached and therapy should be modified if needed. We propose division of the treatment strategies at our disposal to induce remission into three classes: high intensity (HSCT), medium intensitiy (CYC and RTX), and low intensity (AZA and MMF). After obtaining remission, maintenance treatment with AZA or MMF should be started (fig. 2).

Unfortunately, and despite recent advances, in some cases, all the treatments currently at our disposal are unable to significantly change the natural course of this disease.

\section{References}

1 Elhai M, Meune C, Avouac J, et al. Trends in mortality in patients with systemic sclerosis over 40 years: a systematic review and meta-analysis of cohort studies. Rheumatology (Oxford) 2012; 51: 1017-1026.

2 Steen VD, Medsger TA. Changes in causes of death in systemic sclerosis, 1972-2002. Ann Rheum Dis 2007; 66: 940-944.

3 Walker UA, Tyndall A, Czirják L, et al. Clinical risk assessment of organ manifestations in systemic sclerosis: a report from the EULAR Scleroderma Trials And Research group database. Ann Rheum Dis 2007; 66: 754-763.

4 Rubio-Rivas M, Royo C, Simeón CP, et al. Mortality and survival in systemic sclerosis: systematic review and meta-analysis. Semin Arthritis Rheum 2014; 44: 208-219.

5 Bouros D, Wells AU, Nicholson AG, et al. Histopathologic subsets of fibrosing alveolitis in patients with systemic sclerosis and their relationship to outcome. Am J Respir Crit Care Med 2002; 165: 1581-1586.

6 Mouthon L, Berezné A, Brauner M, et al. Pneumopathie infiltrante diffuse de la sclerodermie systemique [Interstitial lung disease in systemic sclerosis]. Rev Mal Respir 2007; 24: 1035-1046.

7 Steen VD, Conte C, Owens GR, et al. Severe restrictive lung disease in systemic sclerosis. Arthritis Rheum 1994 37: 1283-1289.

8 Bagnato GL, Harari S. Cellular interactions in the pathogenesis of interstital lung diseases. Eur Respir Rev 2015; 24: $102-114$

9 Massagué J, Gomis RR. The logic of TGF- $\beta$ signaling. FEBS Lett 2006; 508: 2811-2820.

10 Bhattacharyya S, Kelley K, Melichian DS, et al. Toll-like receptor 4 signaling augments transforming growth factor- $\beta$ responses: a novel mechanism for maintaining and amplifying fibrosis in scleroderma. Am J Pathol 2013; 182: 192-205.

11 Manetti M, Rosa I, Messerini L, et al. A loss of telocytes accompanies fibrosis of multiple organs in systemic sclerosis. J Cell Mol Med 2014; 18: 253-262.

12 Yanaba K, Yoshizaki A, Asano Y, et al. Serum IL-33 levels are raised in patients with systemic sclerosis: association with extent of skin sclerosis and severity of pulmonary fibrosis. Clin Rheumatol 2011; 30: 825-830.

13 Reese C, Perry B, Heywood J, et al. Caveolin-1 deficiency may predispose African Americans to systemic sclerosis-related interstitial lung disease. Arthritis Rheumatol 2014; 66: 1909-1919.

14 De Santis M, Inzitari R, Bosello SL, et al. $\beta$-Thymosins and interstitial lung disease: study of a scleroderma cohort with a one-year follow-up. Respir Res 2011; 12: 22.

15 Lindahl GE, Stock CJ, Shi-Wen X, et al. Microarray profiling reveals suppressed interferon stimulated gene program in fibroblasts from scleroderma-associated interstitial lung disease. Respir Res 2013; 14: 80

16 Christmann RB, Sampaio-Barros P, Stifano G, et al. Association of interferon- and transforming growth factor $\beta$-regulated genes and macrophage activation with systemic sclerosis-related progressive lung fibrosis. Arthritis Rheumatol 2014; 66: 714-725.

17 Assassi S, Wu M, Tan FK, et al. Skin gene expression correlates of severity of interstitial lung disease in systemic sclerosis. Arthritis Rheum 2013; 65: 2917-2927.

18 Zhu H, Luo $\mathrm{H}$, Li Y, et al. MicroRNA-21 in scleroderma fibrosis and its function in TGF- $\beta$-regulated fibrosis-related genes expression. J Clin Immunol 2013; 33: 1100-1109.

19 Hamaguchi Y. Autoantibody profiles in systemic sclerosis: predictive value for clinical evaluation and prognosis. J Dermatol 2010; 37: 42-53.

20 Kostopoulos C, Rassidakis A, Sfikakis PP, et al. Small airways dysfunction in systemic sclerosis. A controlled study. Chest 1992; 102: 875-881.

21 Ungerer RG, Tashkin DP, Furst D, et al. Prevalence and clinical correlates of pulmonary arterial hypertension in progressive systemic sclerosis. Am J Med 1983; 75: 65-74.

22 Tashkin DP, Volkmann ER, Tseng CH, et al. Relationship between quantitative radiographic assessments of interstitial lung disease and physiological and clinical features of systemic sclerosis. Ann Rheum Dis 2014; [In press DOI: 10.1136/annrheumdis-2014-206076].

23 Desai SR, Veeraraghavan S, Hansell DM, et al. CT features of lung disease in patients with systemic sclerosis: comparison with idiopathic pulmonary fibrosis and nonspecific interstitial pneumonia. Radiology 2004; 232: 560-567. 

function, exercise testing, and quality of life. Chest 2007; 131: 672-681.

30 Camiciottoli G, Diciotti S, Bartolucci M, et al. Whole-lung volume and density in spirometrically-gated inspiratory and expiratory CT in systemic sclerosis: correlation with static volumes at pulmonary function tests. Sarcoidosis Vasc Diffuse Lung Dis 2013; 30: 17-27.

31 Picano E, Matucci-Cerinic M. Unnecessary radiation exposure from medical imaging in the rheumatology patient. Rheumatology (Oxford) 2011; 50: 1537-1539.

32 Gargani L, Doveri M, D’Errico L, et al. Ultrasound lung comets in systemic sclerosis: a chest sonography hallmark of pulmonary interstitial fibrosis. Rheumatology (Oxford) 2009; 48: 1382-1387.

33 Barskova T, Gargani L, Guiducci S, et al. Lung ultrasound for the screening of interstitial lung disease in very early systemic sclerosis. Ann Rheum Dis 2013; 72: 390-395.

34 Greidinger EL, Flaherty KT, White B, et al. African-American race and antibodies to topoisomerase I are associated with increased severity of scleroderma lung disease. Chest 1998; 114: 801-807.

35 Assassi S, Sharif R, Lasky RE, et al. Predictors of interstitial lung disease in early systemic sclerosis: a prospective longitudinal study of the GENISOS cohort. Arthritis Res Ther 2010; 12: R166.

36 Plastiras SC, Karadimitrakis SP, Ziakas PD, et al. Scleroderma lung: initial forced vital capacity as predictor of pulmonary function decline. Arthritis Rheum 2006; 55: 598-602.

37 Khanna D, Tseng CH, Farmani N, et al. Clinical course of lung physiology in patients with scleroderma and interstitial lung disease. Arthritis Rheum 2011; 63: 3078-3085.

38 Goh NS, Desai SR, Veeraraghavan S, et al. Interstitial lung disease in systemic sclerosis: a simple staging system. Am J Respir Crit Care Med 2008; 177: 1248-1254.

39 Winstone TA, Assayag D, Wilcox PG, et al. Predictors of mortality and progression in scleroderma-associated interstitial lung disease: a systematic review. Chest 2014; 146: 422-436.

40 Castellví I, Simeón-Aznar CP, Sarmiento M, et al. Association between nailfold capillaroscopy findings and pulmonary function tests in patients with systemic sclerosis. J Rheumatol 2015; 42: 222-227.

41 Lee CG, Herzog EL, Ahangari F, et al. Chitinase 1 is a biomarker for and therapeutic target in scleroderma-associated interstitial lung disease that augments TGF- $\beta 1$ signaling. J Immunol 2012; 189: $2635-2644$.

42 Brissett M, Veraldi KL, Pilewski JM, et al. Localized expression of tenascin in systemic sclerosis-associated pulmonary fibrosis and its regulation by insulin-like growth factor binding protein 3. Arthritis Rheum 2012; 64: $272-280$.

43 Lambrecht S, Smith V, De Wilde K, et al. Growth differentiation factor 15, a marker of lung involvement in systemic sclerosis, is involved in fibrosis development but is not indispensable for fibrosis development. Arthritis Rheum 2014; 66: 418-427.

44 Hesselstrand R, Wildt M, Bozovic G, et al. Biomarkers from bronchoalveolar lavage fluid in systemic sclerosis patients with interstitial lung disease relate to severity of lung fibrosis. Respir Med 2013; 107: 1079-1086.

45 van Bon L, Affandi AJ, Broen J, et al. Proteome-wide analysis and CXCL4 as a biomarker in systemic sclerosis. N Engl J Med 2014; 370: 433-443.

46 De Lauretis A, Sestini P, Pantelidis P, et al. Serum interleukin 6 is predictive of early functional decline and mortality in interstitial lung disease associated with systemic sclerosis. J Rheumatol 2013; 40: 435-446.

47 Tashkin DP, Elashoff R, Clements PJ, et al. Effects of 1-year treatment with cyclophosphamide on outcomes at 2 years in scleroderma lung disease. Am J Respir Crit Care Med 2007; 76: 1026-1034.

48 Hoyles RK, Ellis RW, Wellsbury J, et al. A multicenter, prospective, randomized, double-blind, placebo-controlled trial of corticosteroids and intravenous cyclophosphamide followed by oral azathioprine for the treatment of pulmonary fibrosis in scleroderma. Arthritis Rheum 2006; 54: 3962-3970.

49 Nadashkevich O, Davis P, Fritzler M, et al. A randomized unblinded trial of cyclophosphamide versus azathioprine in the treatment of systemic sclerosis. Clin Rheum 2006; 25: 205-212.

50 Guillevin L, Cordier JF, Lhote F, et al. A prospective, multicenter, randomized trial comparing steroids and pulse cyclophosphamide versus steroids and oral cyclophosphamide in the treatment of generalized Wegener's granulomatosis. Arthritis Rheum 1997; 40: 2187-2198.

51 Iudici M, Cuomo G, Vettori S, et al. Low-dose pulse cyclophosphamide in interstitial lung disease associated with systemic sclerosis (SSc-ILD): efficacy of maintenance immunosuppression in responders and non-responders. Semin Arthritis Rheum 2015; 44: 437-444.

52 Airò $\mathrm{P}$, Danieli E, Rossi M, et al. Intravenous cyclophosphamide for interstitial lung disease associated to systemic sclerosis: results with an 18-month long protocol including a maintenance phase. Clin Exp Rheumatol 2007; 25: 293-296.

53 Giacomelli R, Valentini G, Salsano F, et al. Cyclophosphamide pulse regimen in the treatment of alveolitis in systemic sclerosis. J Rheumatol 2002; 29: 731-736.

54 Simeón-Aznar CP, Fonollosa-Pla V, Tolosa-Vilella C, et al. Effect of mycophenolate sodium in scleroderma-related interstitial lung disease. Clin Rheumatol 2011; 30: 1393-1398.

55 Gerbino AJ, Goss CH, Molitor JA. Effect of mycophenolate mofetil on pulmonary function in scleroderma-associated interstitial lung disease. Chest 2008; 133: 455-460.

56 Koutroumpas A, Ziogas A, Alexiou I, et al. Mycophenolate mofetil in systemic sclerosis-associated interstitial lung disease. Clin Rheumatol 2010; 29: 1167-1168.

57 Liossis SNC, Bounas A, Andonopoulos A. Mycophenolate mofetil as first-line treatment improves clinically evident early scleroderma lung disease. Rheumatology 2006; 45: 1005-1008. 
58 Zamora AC, Wolters PC, Collard HR, et al. Use of mycophenolate mofetil to treat scleroderma-associated interstitial lung disease. Respir Med 2008; 102: 150-155.

59 Tzouvelekis A, Galanopoulos N, Bouros E, et al. Effect and safety of mycophenolate mofetil or sodium in systemic sclerosis-associated interstitial lung disease: a meta-analysis. Pulm Med 2012; 2012: 143637.

60 Panopoulos ST, Bournia VK, Trakada G, et al. Mycophenolate versus cyclophosphamide for progressive interstitial lung disease associated with systemic sclerosis: a 2-year case control study. Lung 2013; 191: 483-489.

61 Walker KM, Pope J, Scleroderma Clinical Trials Consortium et al. Expert agreement on EULAR/EUSTAR recommendations for the management of systemic sclerosis. J Rheumatol 2011; 38: 1326-1328.

62 Paone C, Chiarolanza I, Cuomo G, et al. Twelve-month azathioprine as maintenance therapy in early diffuse systemic sclerosis patients treated for 1-year with low dose cyclophosphamide pulse therapy. Clin Exp Rheumatol 2007; 25: 613-616.

63 Bérezné A, Ranque B, Valeyre D, et al. Therapeutic strategy combining intravenous cyclophosphamide followed by oral azathioprine to treat worsening interstitial lung disease associated with systemic sclerosis: a retrospective multicenter open-label study. Rheumatol 2008; 35: 1064-1072.

64 Griffiths B, Miles S, Moss $\mathrm{H}$, et al. Systemic sclerosis and interstitial lung disease: a pilot study using pulse intravenous methylprednisolone and cyclophosphamide to assess the effect on high resolution computed tomography scan and lung function. J Rheumatol 2002; 29: 2371-2378.

65 Yiannopoulos G, Pastromas V, Antonopoulos I, et al. Combination of intravenous pulses of cyclophosphamide and methylprednisolone in patients with systemic sclerosis and interstitial lung disease. Rheumatol Int 2007; 27: 357-361.

66 Helfrich DJ, Banner B, Steen VD, et al. Normotensive renal failure in systemic sclerosis. Arthritis Rheum 1989; 32: $1128-1134$

67 DeMarco PJ, Weisman MH, Seibold JR, et al. Predictors and outcomes of scleroderma renal crisis: the high dose versus low-dose of penicillamine in early diffuse systemic sclerosis trial. Arthritis Rheum 2002; 46: 2983-2989.

68 Daoussis D, Liossis SN, Tsamandas AC, et al. Experience with rituximab in scleroderma: results from a 1-year, proof-of-principle study. Rheumatology 2010; 49: 271-280.

69 Daoussis D, Liossis SN, Tsmandas AC, et al. Effect of long-term treatment with rituximab on pulmonary function and skin fibrosis in patients with diffuse systemic sclerosis. Clin Exp Rheum 2012; 30: Suppl. 71, S17-S22.

70 Jordan S, Distler JH, Maurer B, et al. Effects and safety of rituximab in systemic sclerosis: an analysis from the European Scleroderma Trial and Research (EUSTAR) group. Ann Rheum Dis 2015; 74: 1188-1194.

71 Distler JH, Jordan S, Airo P, et al. Is there a role for TNF $\alpha$ antagonists in the treatment of SSc? EUSTAR expert consensus development using the Delphi technique. Clin Exp Rheumatol 2011; 29: Suppl. 65, S40-S45.

72 Distler JHW, Distler O. Intracellular tyrosine kinases as novel targets for anti-fibrotic therapy in systemic sclerosis. Rheumatology 2008; 47: Suppl. 5, 10-11.

73 Spiera RF, Gordon JK, Mersten JN, et al. Imatinib mesylate (Gleevec) in the treatment of diffuse cutaneous systemic sclerosis: results of a 1-year, phase IIa, single-arm, open-label clinical trial. Ann Rheum Dis 2011; 70: 1003-1009.

74 Khanna D, Saggar R, Mayes MD, et al. A one-year, phase I/IIa, open-label pilot trial of imatinib mesylate in the treatment of systemic sclerosis-associated active interstitial lung disease. Arthritis Rheum 2011; 63: 3540-3546.

75 Pope J, McBain D, Petrlich L, et al. Imatinib in active diffuse cutaneous systemic sclerosis: results of a six-month, randomized, double-blind, placebo-controlled, proof-of-concept pilot study at a single center. Arthritis Rheum 2011; 63: 3547-3551.

76 Fraticelli P, Gabrielli B, Pomponio G, et al. Low-dose oral imatinib in the treatment of systemic sclerosis interstitial lung disease unresponsive to cyclophosphamide: a phase II pilot study. Arthritis Res Ther 2014; 16: R144.

77 Daniels CE, Lasky JA, Limper AH, et al. Imatinib treatment for idiopathic pulmonary fibrosis: randomized placebo-controlled trial results. Am J Respir Crit Care Med 2010; 181: 604-610.

78 Zhao L, Xiao K, Wang $\mathrm{H}$, et al. Thalidomide has a therapeutic effect on interstitial lung fibrosis: evidence from in vitro and in vivo studies. Clin Exp Immunol 2009; 157: 310-315.

79 Takehara K, Ihn H, Sato S. A randomized, double-blind, placebo-controlled trial: intravenous immunoglobulin treatment in patients with diffuse cutaneous systemic sclerosis. Clin Exp Rheumatol 2013; 31: 151-156.

80 Levy Y, Amital H, Langevitz P, et al. Intravenous immunoglobulin modulates cutaneous involvement and reduces skin fibrosis in systemic sclerosis: an open-label study. Arthritis Rheum 2004; 50: 1005-1007.

81 Amital H, Rewald E, Levy Y, et al. Fibrosis regression induced by intravenous gammaglobulin treatment. Ann Rheum Dis 2003; 62: 175-177.

82 Nacci F, Righi A, Conforti ML, et al. Intravenous immunoglobulins improve the function and ameliorate joint involvement in systemic sclerosis: a pilot study. Ann Rheum Dis 2007; 66: 977-979.

83 Mauhin W, Rivière S, Cabane J, et al. Improvement in lung fibrosis using intravenous immunoglobulin in systemic sclerosis with myositis. Scand J Rheumatol 2014; 43: 170-171.

84 Tyndall A, Matucci-Cerinic M. Haematopoietic stem cell transplantation for the treatment of systemic sclerosis and other autoimmune disorders. Expert Opin Biol Ther 2003; 3: 1041-1049.

85 van Laar JM, Farge D, Sont JK, et al. Autologous hemtopoietic stem cell transplantation vs intravenous pulse cyclophosphamide in diffuse cutaneous systemic sclerosis: a randomized clinical trial. JAMA 2014; 311: 2490-2498.

86 Khan IY, Singer LG, de Perrot M, et al. Survival after lung transplantation in systemic sclerosis. A systematic review. Respir Med 2013; 107: 2081-2087.

87 Shitrit D, Amital A, Peled N, et al. Lung transplantation in patients with scleroderma: case series, review of the literature, and criteria for transplantation. Clin Transplant 2009; 23: 178-183.

88 Schachna L, Medsger TA Jr, Dauber JH, et al. Lung transplantation in scleroderma compared with idiopathic pulmonary fibrosis and idiopathic pulmonary arterial hypertension. Arthritis Rheum 2006; 54: 3954-3961.

89 Saggar R, Khanna D, Furst DE, et al. Systemic sclerosis and bilateral lung transplantation: a single centre experience. Eur Respir J 2010; 36: 893-900.

90 Massad MG, Powell CR, Kpodonu J, et al. Outcomes of lung transplantation in patients with scleroderma. World J Surg 2005; 29: 1510-1515.

91 Launay D, Savale L, Berezne A, et al. Lung and heart-lung transplantation for systemic sclerosis patients. A monocentric experience of 13 patients, review of the literature and position paper of a multidisciplinary Working Group. Presse Med 2014; 43: e345-e363. 